Meta

Journal des traducteurs

Translators' Journal

\title{
Point de vue sur la définition
}

\section{Élisabeth Blanchon}

Volume 42, numéro 1, mars 1997

Lexicologie et terminologie

URI : https://id.erudit.org/iderudit/002090ar

DOI : https://doi.org/10.7202/002090ar

Aller au sommaire du numéro

Éditeur(s)

Les Presses de l'Université de Montréal

ISSN

0026-0452 (imprimé)

1492-1421 (numérique)

Découvrir la revue

Citer cet article

Blanchon, É. (1997). Point de vue sur la définition. Meta, 42(1), 168-173.

https://doi.org/10.7202/002090ar

\section{Résumé de l'article}

Dans cet article, on démontre la nécessité pour les terminologues d'élaborer des méthodes précises de rédaction des définitions. On voit en quoi la définition est utile en terminologie, quelle est la structure de la définition et quels sont les modèles à respecter. Enfin, on traite du problème de l'adéquation au public. 


\title{
POINT DE VUE SUR LA DÉFINITION
}

\author{
ÉLISABETH BLANCHON \\ Université Paris Nord-Paris XIII, Villetaneuse, France
}

\begin{abstract}
Résumé
Dans cet article, on démontre la nécessité pour les terminologues d'élaborer des méthodes précises de rédaction des définitions. On voit en quoi la définition est utile en terminologie, quelle est la structure de la définition et quels sont les modèles à respecter. Enfin, on traite du problème de l'adéquation au public.
\end{abstract}

\begin{abstract}
This article stresses the necessity for terminologists to establish a rigorous methodology for writing definitions. It deals with the usefulness of definitions in terminology, their structure, models to be observed, and raises the issue of suitability for the general public.
\end{abstract}

\section{INTRODUCTION}

La définition constitue l'un des problèmes les plus épineux que rencontrent les terminologues. Un premier point, éludé la plupart du temps, est celui de son utilité, remise en question par certains, dont on peut tenter de comprendre la position. Un autre aspect est celui de la structure de la définition et des modèles à respecter. Assurément, les manuels de terminologie décrivent les différents types de définitions et fournissent des règles d'élaboration. Mais au bout du compte, ces règles, qui concernent le plus souvent la forme, renvoient le terminologue à son expérience et sont difficilement applicables telles quelles, ce qui est manifeste quand on se trouve en situation d'enseignement. Enfin, le problème de l'adéquation au public, assez peu étudié en lexicographie générale, l'est encore moins en langue de spécialité. On a tendance à faire comme si la terminologie était destinée à la communication entre spécialistes, et à rédiger les définitions dans cette seule perspective. Or, si tel est parfois le cas, ce n'est sûrement pas le plus fréquent.

\section{LA DÉFINITION EST-ELLE UTILE?}

Nous reprenons ici le titre un peu provocateur d'une communication de Franz Josef Hausmann. L'auteur faisait certes surtout référence aux définitions des dictionnaires d'apprentissage, mais la question peut se poser aussi en terminologie.

Certains terminologues estiment que «la définition n'est parfois justifiée qu'en vertu de la tradition qui en fait l'un des éléments clés de la lexicographie». C'est la position de Daniel Gouadec, par exemple. Robert Dubuc dit ainsi que «sous l'influence de la lexicographie», on a peut-être trop «déifié» la nécessité de la définition. Il privilégie quant à lui le contexte et l'idée d'un crochet terminologique permettant d'apparier les termes dans différentes langues. Ce crochet terminologique s'établit grâce à «l'analyse du contenu notionnel du contexte», qui «doit viser à dégager les éléments significatifs de la notion sans que ces éléments débouchent nécessairement sur l'élaboration d'une définition en bonne et due forme». La définition lui semble parfois dangereuse en ce qu'elle risque de figer la notion qu'elle structure et donc d'empêcher la terminologie de «se tenir constamment en résonance avec l'usage», qui, lui, n'est par essence pas figé.

Il faut souligner que ces deux auteurs travaillent surtout dans le cadre de la terminologie pour la traduction, peut-être plus proche de la tradition de la lexicographie bilingue, 
généralement dépourvue de définition. Dans ce cadre-là, surtout si c'est la même personne qui traduit et qui élabore sa terminologie (cas le plus fréquent en France), il lui est difficile, sinon impossible, pour des raisons de rentabilité, de rédiger des définitions en bonne et due forme. Elle n'en a tout simplement pas le temps.

En outre, si le terminologue a effectivement besoin d'une définition pour situer la notion dans un système notionnel, tel n'est peut-être pas le cas pour le traducteur, qui cherche avant tout à vérifier l'équivalence des termes.

D'autres contournent cet épineux problème du choix de la définition ou du contexte en ne retenant que des définitions toutes prêtes. Par exemple, il semble que le Secrétariat d'État du Canada conseille à ses terminologues de se rabattre toujours sur des définitions déjà rédigées ou sur des contextes définitoires. Ainsi, dans un cadre d'enseignement peuton demander à des étudiants de terminologie de fournir deux définitions pour chaque terme, comme le fait par exemple Herbert Eisele à I'ISIT.

Cette solution n'est pas sans défauts. On peut notamment se poser la question de l'identité de la notion décrite par deux définitions différentes. Elle est peut-être symptomatique de la difficulté que l'on rencontre pour formaliser la manière de rédiger à coup sûr une bonne définition et a fortiori pour enseigner l'art de la définition. Mais n'est-ce pas une façon de baisser les bras?

Contexte et définition apportent des informations différentes. Le contexte peut en effet ne fournir que des informations incomplètes sur la notion, ce qui peut induire un certain flou notionnel nuisant à la compréhension. La définition, au contraire, est censée faire le tour de la notion, dans un environnement et dans un but donné, et bien la situcr dans le système notionnel du domaine. Encore faut-il dire que dans ce cas, abondance de biens peut nuire, et que le fait de disposer de deux définitions ou plus risque d'engendrer le même flou notionnel.

Des définitions, bonnes ou mauvaises, ne sont pas toujours disponibles: l'un des rôles de la terminologie est précisément de combler les lacunes des dictionnaires, et les ouvrages spécialisés dans un domaine ne prennent pas toujours la peine de définir les notions qu'ils manient.

Enfin, ces définitions toutes prêtes sont extraites d'un tout cohérent pour être réutilisées dans un tout autre système de définitions. Restent-elles utilisables sans retouches dans ce nouvel environnement (les termes employés sont-ils bien définis dans le nouveau produit terminologique, par exemple)? Doit-on les réécrire entièrement pour les rendre cohérentes avec le reste du travail ? Dans ce cas, on perd tout le bénéfice qu'apporte cette méthode : le temps gagné par le fait de les emprunter est perdu à nouveau pour la réécriture. Ou choisit-on de faire fi de toutes les recommandations en matière de définition? Enfin, si l'on emprunte des définitions de spécialistes, il faut tenir compte du fait qu'ils ont probablement chacun leur façon de décrire un concept, en le situant dans une hiérarchie qui reflète leur approche personnelle d'un domaine, que chacun structure à sa manière. Ce qui pose à nouveau le problème de la cohérence définitionnelle du produit final.

À la question initiale de l'utilité de la définition, il faut répondre oui. En fait, tout un chacun est intimement convaincu du caractère fondamental de la définition : elle est utile au traducteur pour être sûr de l'équivalence de termes, elle est utile au terminologue pour constituer son système notionnel, elle est utile au documentaliste pour contrôler son thésaurus, à l'étudiant pour maîtriser la notion, au professeur pour la faire comprendre, au juriste pour servir de point d'appui. Ses détracteurs, s'ils en sont vraiment, ne le sont que par souci d'efficacité, d'économie. Ils le sont peut-être aussi parce qu'ils ont trop souvent été déçus par de mauvaises définitions ou par des définitions mal adaptées à leurs besoins, à leurs attentes, et trop fréquentes, il faut bien le dire. Car la définition est utile, mais quelle définition? 


\section{QUEL TYPE DE DÉFINITION ?}

La plupart des manuels de terminologie ou les études consacrées à la définition, tant en lexicographie qu'en terminologie, procèdent d'une démarche apparemment parfaitement logique et satisfaisante. En effet, ils proposent tout d'abord une partie purement descriptive et théorique, avec une typologie des différents types de définitions possibles (typologie qui varie d'ailleurs selon le point de vue des auteurs), puis une partie prescriptive dans laquelle ils énumèrent un certain nombre de règles et de principes à suivre pour arriver à une bonne définition. Que l'on aimerait que les choses soient aussi simples!

On trouve ainsi, pour bien marquer les différences entre la lexicographie traditionnelle et la terminologie, une première opposition entre :

- définition lexicographique, définition encyclopédique, équivalente ou distincte de la définition terminologique (Uzoma Chukwu);

définition linguistique, définition ontologique, définition terminologique (Teresa Cabré) ;

- définition nominale, définition ostensive, définition conceptuelle (Ingetraut Dahlberg).

Un deuxième axe concerne le contenu logique des définitions terminologiques:

- définition par compréhension ou spécifique, définition par extension ou générique (Normes ISO, par exemple);

- définition générique, définition partitive, définition par opposition (condamnée), définition fonctionnelle, opérationnelle ou génétique (Ingetraut Dahlberg).

Une troisième opposition relève plus de la structure des définitions:

- définition métalinguistique, paraphrastique, hyperonymique, métonymique, dérivationnelle, approximative, synonymique, positive, négative, conjonctionnelle (Robert Martin);

- définition par analyse (genus et differentia), par synonyme, par paraphrase, par synthèse (identification des relations, description), par implication (utilisation du mot dans un contexte explicatif), définition par dénotation (liste d'exemples, extension), définition par démonstration (définition ostensive), définitions mixtes, par analyse et description, par synonyme et description, par synonyme et analyse, (Juan Carlos Sager);

- Définition référentielle, définition à lieurs, définition métalinguistique, définition synonymique, définition par dérivation (Agnès Tutin).

Bien sûr, dans leur partie prescriptive, ces travaux donnent des règles quant à l'organisation logique du contenu, à son choix et à ses limites. Mais ces règles, qui sont les plus fondamentales, sont aussi les plus générales.

Partout on rappelle que la définition doit situer clairement et immédiatement la notion dans un système notionnel, c'est-à-dire, nous dit la norme ISO 704,

la décrire à un niveau d'abstraction donné, distinguer la notion des notions apparentées, établir des relations entre la notion en cause et les autres notions du système afin de déterminer la place qu'occupe cette notion à l'intérieur du système. [...] La définition doit contenir les caractères essentiels de la notion représentant un objet particulier. Les caractères extrinsèques et intrinsèques peuvent jouer un rôle de caractères essentiels.

Bien sûr cette norme est illustrée d'exemples, mais cela reste bien théorique, et ce l'est à peine moins dans les manuels. 
Même dans un ouvrage à orientation aussi pratique que celui de Daniel Gouadec, qui préconise «d'adopter une norme de construction des définitions», on se trouve renvoyé à des critères abstraits, théoriques, certes abondamment commentés et étayés d'exemples, mais demeurant quand même assez généraux. «La définition fait se succéder, dans cet ordre, (1) l'éventuelle stipulation de 'sur-limites' de champ, (2) la nature du référent, (3) la localisation du référent, (4) le générique (intégrateur) et/ou les spécifiques (composants) du référent, et (5) les caractères définitoires du référent, dont sa fonction.» (Gouadec)

Des règles plus précises sont édictées à propos de la structure physique de la définition. On en a une abondance, dont certaines sont le reflet direct d'un choix théorique.

- le défini ne doit pas faire partie de la définition;

- la définition doit avoir la même forme grammaticale que le terme;

- la définition ne doit pas commencer par un article;

- la définition ne doit pas être métalinguistique (ou métaterminogique);

- la définition doit être constituée d'une seule phrase.

Le respect de ces règles pratiques ne garantit en rien la qualité d'une définition. Nous ne gagnerons pas le paradis terminologique par l'achat d'indulgences typographiques.

On aimerait croire qu'il est possible d'aller plus loin dans la formalisation des règles définitionnelles concernant le contenu. La tentative d'Igor Mel`čuk est certes intéressante mais peut-être est-elle pour le coup trop ambitieuse.

\section{L'ADÉQUATION AU PUBLIC}

Pour qu'une terminologie soit utile, il est indispensable, tout un chacun l'admet, que les définitions qu'elle contient soient élaborées en tenant compte du public que vise le produit terminologique dans lequel elle s'insère. Et l'on citera ce que Claude Dubois disait de la définition lexicographique au Colloque de Pointe-au-Pic en 1977: "La définition est aussi fonction de la cible du dictionnaire, du type de lecteur auquel elle s'adresse. Les lexicographes doivent adapter la paraphrase synonymique scientifique afin de la transcoder dans une forme écrite capable d'être reçue par le groupe socioculturel qu'ils ont défini comme étant le récepteur potentiel de l'information contenue dans le dictionnaire.» Il faut que la définition évoque quelque chose pour le public et corresponde à son univers de croyance, car, comme le souligne très clairement Robert Martin : «L'univers du locuteur dépend des informations qu'il possède, des connaissances acquises, des faits mémorisés. On comprend ainsi qu'une même proposition (p. ex. L'atome est formé d'un noyau et d'électrons) puisse avoir, selon le degré de culture, d'information ou de savoir, des contenus plus ou moins précis.»

La définition sera nécessaircment différente selon qu'on s'adresse à un public d'étudiants d'un domaine, de spécialistes, de traducteurs, ou de ceux que Chukwu appelle les «terminologues-linguistes», qu'il distingue des «terminologues-experts» comme abordant la terminologie par le biais de la linguistique et donc comme ne maîtrisant pas la connaissance technique d'un domaine (ce qui n'est guère différent de la situation du terminologue débutant ou du terminologue qui aborde un nouveau domaine). En outre «la définition ne présente jamais qu'un intérêt anecdotique pour le spécialiste ou professionnel» (Gouadec). Mais qui sont les plus gros consommateurs de terminologie? Dans sa récente communication au colloque TKE'93, «Role and Representation of Terminological Definitions», Gerhard Rahmstorf identifiait huit catégories différentes d'utilisateurs de définitions:

- l'utilisateur de base, l'étudiant, etc.;

- le traducteur;

- le scientifique, l'ingénieur, le chercheur; 
- le spécialiste de normalisation, le terminologue;

- le spécialiste de l'information, l'indexeur, le spécialiste de l'interrogation;

- l'épistémologue, le psychologue;

- l'ingénieur de la connaissance;

le linguiste.

Cette typologie est certes intéressante, d'autant plus que les catégories d'utilisateurs sont accompagnées d'une mention du centre d'intérêt principal de l'utilisateur concerné, et de la fonction que la définition remplit pour lui, mais on a l'intuition qu'il manque certains types d'utilisateurs (peut-être par exemple les juristes) et, plus gênant, ces catégories ont été, de l'aveu même de l'auteur, définies a priori.

La norme ISO 704 : Principes et méthodes de la terminologie, précise que l'on doit mentionner, dans la définition, «la notion générique la plus proche (le genre), qui est soit déjà définie, soit supposée généralement connue». Elle ajoute que «le spécialiste qui se charge d'élaborer une définition doit s'assurer que tous les termes seront compris correctement».

Reste à déterminer comment on peut vérifier qu'une notion est généralement connue, comment on peut adapter une définition à un public. Or ce problème, s'il est mentionné par les manuels de terminologie, reste sans réponse précise.

C'est peut-être l'Office de la langue française qui est allé le plus loin sur ce point. En effet, dans sa politique d'implantation terminologique, l'office se soucie désormais d'adapter ses produits terminologiques aux groupes visés, ce qui implique une réflexion sur le type de support, sur le choix des termes et sur la définition. En effet, comme le souligne Christiane Loubier: «Les produits terminologiques ne sont pas tous destinés à des spécialistes qui ont une grande connaissance du système notionnel de leur domaine. Ainsi les définitions peuvent être différentes selon la clientèle et c'est l'usage qui doit en être fait qui va déterminer leur degré de technicité». Mais, là encore, on aimerait disposer de critères précis.

En outre, il est difficile d'aborder cette difficulté de manière totalement autonome, sans évoquer en même temps d'autres problèmes délicats, car le choix d'un public influe aussi sur le choix des termes: quelles limites doit-on fixer à la nomenclature et, partant, à la circularité du dictionnaire? Quel niveau de langue va-t-on choisir? Car il serait illusoire de penser, même si cela a fait l'objet de peu d'études à notre connaissance, que les langues de spécialités échappent à ce phénomène et que l'ouvrier utilise les mêmes termes que l'ingénieur. Quelle organisation notionnelle choisir pour un domaine, car cela diffère selon les individus, leur formation, leur point de vue, etc. Une structuration particulière d'un domaine va se refléter dans un choix bien précis de notion superordonnée et de caractères spécifiques (voir l'exemple célèbre de l'eau en physique ou de l'eau en chimie, Norme ISO 704). Selon la formation du public, on aura peut-être intérêt à ne pas choisir la notion immédiatement supérieure comme point d'ancrage de la définition, mais une notion plus générique et plus immédiatement accessible.

Un certain nombre de travaux sont menés actuellement pour tenter d'extraire automatiquement les informations sémantiques présentes dans les dictionnaires, et donc essentiellement dans les définitions. On peut citer les travaux du Centre national d'Étude des télécommunications (CNET-Lannion, France), du Centre scientifique d'IBM-Allemagne, à Heidelberg, ou celui, plus ancien, du laboratoire d'informatique de l'Université de Cambridge. Ils se heurtent tous à ce qu'Ingetraut Dahlberg avait déjà souligné en 1982, à la suite de l'étude de 300 dictionnaires monolingues allemands, «an almost chaotic situation with regard to form and structure of terminological definitions». L'absence de régularité des définitions constitue la pierre d'achoppement de ces travaux. On peut espérer que 
leurs analyses permettront d'alimenter une réflexion en amont, pour obliger les terminologues à élaborer enfin des méthodes précises de rédaction des définitions. La tâche est immense pour tenter d'y voir plus clair et de forger des outils adéquats.

\section{RÉFÉRENCES}

CABRÉ, Teresa (1992) : La terminologia. La teoria, els mètodes, les aplicacions, Barcelone, Editorial Empúriex.

CHUKWU, Uzoma Everest (1993): Le repérage des termes dans un corpus bilingue anglais / français, thèse de doctorat, Université Lumière-Lyon 2.

DE BESSÉ, Bruno (1990): «La définition terminologique», Actes du Colloque La définition, organisé par le CELEX (Centre d'Études du Lexique) de l'Université Paris-Nord (Paris XIII, Villetaneuse) à Paris, les 18 et 19 novembre 1988, Paris, Larousse.

DUBOIS, Claude (1979): «La spécificité de la définition en terminologie (par comparaison avec la définition en lexicographie)», Actes du sixième colloque international de terminologie, Pointe-au-Pic (Québec), du 2 au 6 octobre 1977, OLF, Éditeur officiel du Québec.

DUBUC, Robert (1992): Manuel pratique de terminologie, $3^{\mathrm{c}}$ édition entièrement revue et mise à jour, Brossard, Linguatech.

DUQUET-PICARD, Diane (1982) : «La définition en terminologie : aspect didactique», Travaux de terminologie, $\mathrm{n}^{\circ}$ 2, GIRSTERM, Université Laval (Québec).

FELBER, Helmut (1987): Manuel de terminologie, Paris, Unesco.

GOUADEC, Daniel (1990): Terminologie, Constitution des données, Paris, Afnor gestion.

HAUSMANN, Franz Josef (1990): «La définition est-elle utile?», Actes du Colloque La définition, organisé par le CELEX (Centre d'Études du Lexique) de l'Université Paris-Nord (Paris XIII, Villetaneuse) à Paris, les 18 et 19 novembre 1988, Paris, Larousse.

ISO Norme $704:$ Principes et méthodes de la terminologie.

ISO Norme 1087 : Vocabulaire de la terminologie.

ISO Norme 10241 (1992): Normes terminologiques internationales-Élaboration et présentation.

ISO Recommandation 860 : Unification internationale des notions et des termes.

LAGACÉ, J. (1984) : «La définition terminologique», Travaux de terminologie, $\mathrm{n}^{\circ} 3$, GIRSTERM, Université Laval (Québec).

LOUBIER, Christiane (1991) : «La terminologie au service de l'implantation», Terminogramme, $\mathrm{n}^{\circ} 57$, Québec, OLF.

MEYER, Ingrid, BOWKER, Lynne et Karen ECK (1991): «Constructing a Knowledge-Based Term Bank : Fundamentals and Implications», Actes du symposium international Terminologie et documentation dans la communication spécialisée, Montréal, Infoterm, Secrétariat d'État du Canada.

MEYER, Ingrid, BOWKER, Lynne et Karen ECK (1992) : «COGNITERM: An Experiment in Building a Terminological Knowledge Base», Proceedings of the Fifth Euralex International Congress, Tampere, Finland, 4-9 août 1992 .

PICHT, Heribert et Jennifer DRASKAU (1985): Terminology, an Introduction, University of Surrey, The Copenhage School of Economics

RAHMSTORF, Gerhard (1993): «Role and Representation of Terminological Definitions», Klaus-Dirk Schmitz (Ed.), TKE'93: Terminology and Knowledge Engineering, Francfort, Indeks Verlag.

REY, Alain (1979) : La terminologie, noms et notions, Paris, PUF, coll. «Que Sais-je ?».

REY, Alain (1990): «Polysémie du terme définition», Actes du Colloque La définition, organisé par le CELEX (Centre d'Études du Lexique) de l'Université Paris-Nord (Paris XIII, Villetaneuse) à Paris, les 18 et 19 novembre 1988 , Paris, Larousse.

REY-DEBOVE, Josette (1978) : Le Métalangage, Paris, Le Robert.

RONDEAU, Guy (1984) : Introduction à la terminologie, $2^{e}$ édition, Chicoutimi (Canada), Gaétan Morin.

SAGER, Juan C. (1990): A Practical Course in Terminology Processing, Amsterdam, John Benjamins.

TUTIN, Agnès (1993) : «Le dictionnaire de langue comme langue de spécialité», Actes de la quatrième journée ERLA-GLAT, Brest, UBO-ENST. 